\title{
Fit Forms and Free Forms of the Masonry Dome
}

\author{
Emanuel Jannasch ${ }^{1}$
}

\begin{abstract}
That tensile hoop stresses will cause an un-chained masonry dome to burst is well known, but the stabilizing influence of hoop-wise compression is rarely discussed. An arch cannot exhibit hoop stresses of any kind, so freestanding masonry arches must fit their own funiculars. But hoop compression does arise in some domes, such as those designed on shallow circular arcs. And as long as the hoop stresses they develop are exclusively compressive (except, of course, at the base), masonry domes are free to take certain non-funicular forms. To explore this design freedom, the author and his students built an unusual array of domes of un-bound and un-mortared masonry. Notable examples include the anticlastic or bell-shaped roofs we call pseudomes, some antidomes that descend from their foundation ring to form basins, and a hemi-toroidal ambidome that rises conventionally from its foundation to a circular crown whence it descends to a pendant oculus. To the best of our knowledge, the antiand ambidomes are unprecedented in the history and theory of structures. The material success of these counterintuitive structures advanced our understanding of masonry, it raised some questions about the natural and the artificial in structural form-finding, and it challenged our preconceptions about the optimal, the ideal, and the free in structural form.
\end{abstract}

Keywords Masonry domes · Funicular · Non-funicular · Catenary · Shell

Emanuel Jannasch

jannasch@dal.ca

1 Dalhousie University School of Architecture, Halifax, Canada 


\section{Introduction}

To describe a dome's form as the rotation of an arch seems reasonable enough, but to imagine its structural action in this way, as a circular array of arches intersecting at the apex, is misleading. The arch-like or meridional action of a masonry dome is not unimportant, but it is far from exclusive. For one thing, it ignores the condition of oculi and lanterns, so obvious in the western tradition. These demonstrate that a dome can stand in the total absence of anything like a keystone. Some authors, for instance Sandaker et al. (2011: 350-356) do acknowledge this logical gap, and portray a radial set of half-arches contained by a tension ring at the bottom and leaning into a compression ring at the top. But reality is seldom as tidy as this image. Ring compression is not restricted to the uppermost course of a dome. Picturing a dome being built with minimal centering, we see that each course completed in its turn takes the position of the upper compression ring. And there is no reason for the hoop compression in any one course to disappear just because one or some other number of courses has been added. Some level of compression will remain in many of the upper courses, even in the completed dome, whether it includes an oculus or not. Each of these courses will thus function as a fully circular arch. All things considered, it could be more coherent and instructive to consider a dome not as the horizontal rotation of a (typically incomplete) vertical arch but as the vertical stacking of complete horizontal arches.

This was the premise taken up by a team of twelve at the Dalhousie University School of Architecture over a two-week period in July of 2014. Student members were Marc Dainow, Zac Jardak, Kirsten Holmlund, Benny Kwok, Elijah Lukac, Lachlan Macdonald, Dan Maj, Brent Schmidt, Ben Weiss, and Mark White, and our software consultant was Naryn Davar. Together we built some unusual and possibly novel dome forms. Not all of them appear to be useful, but together they challenged our preconceptions about masonry construction. In regions where tensile materials are costly and employment is desirable, and especially where there is seismic risk, a better understanding of masonry structures is of immediate human value. We also agreed that having to re-think our ideas of dome behavior encouraged us to examine and refine our mental models of other structural forms. Initial results were presented in 2016 at the 3d International Conference on Structures and Architecture in Guimarães, Portugal (Jannasch 2016a). Response in this forum encouraged us to address a wider audience. This paper recapitulates our basic findings, provides further examples and details, and features better images. The structural analysis here is also more complete.

The paper is organized on the basis of structural form. The first two sections revisit the conventional understanding of funicular design: first in the matter of catenary arches, then in the idea of the funicular dome. The remainder of the paper considers some unusual and distinctly non-funicular dome forms. Some of them appear-however fleetingly-in the history of structures, others we conceived and built. The third section of the paper covers a class of structure we call hyperfuniculars. These can be understood as radically compressive structures, benefitting from hoop-wise as well as meridional compression. The last section is on what we 
termed a-funicular domes, which bring several actions into explicit play besides compression. These are the least conventional structures, but the array of behaviors they exhibit are implicit in all masonry domes.

The two weeks of work also gave food for thought on the nature of artifice and related topics. Ruminations on form-finding, naturalness, freedom, and fitness arise throughout the text. Our questions on these subjects are gathered together in the conclusions, though perhaps not answered conclusively.

\section{The Catenary and its Relatives}

\section{Varieties of the Thin Arch}

Translations vary, but Hooke's centuries-old principle that "as hangs a flexible continuum, so, inverted, stands a contiguity of rigid parts" remains a cornerstone of structural thinking. The dictum is generally taken to mean that the curve defined by a uniform free-hanging chain is the same curve that is required in a uniformly thin, freestanding, masonry arch. But there are few freestanding masonry arches in the world, and these often thicken toward the base for better bearing and stability. And in fact, there is nothing in Hooke's expression that excludes external loads or that limits the hanging element to a constant cross section. Given Hooke's practical bent and his extraordinary insight, we might guess that he had the broader case of the funicular in mind, which responds to any combination of internal and external loads, and of which the plain mathematical catenary is a rare and rarified example. Whatever the history, it is important to distinguish between the two terms. Here we will use the term catenary to indicate the curve of any freely hanging chains or their compressive analogues, including those of variable section. The term funicular will be used for the more general case in which applied loads may be added to the selfweights.

\section{Elasticity and Equal Stress in Nature and in Artifact}

The standard or plain catenary, as we call it here, models a long, heavy, inelastic chain. Replacing the chain with a spring transforms the curve. The spring will distend as axial loads accumulate toward the anchor points while self-weight remains more concentrated toward mid-span. The result is an elastic catenary, a curve that is pointier at the trough than its plain cousin, and less steeply inclined at the anchorages. It is worth noting that this curve does not invert to a compressive counterpart. If we want to build an arch of elastically compressible materials, we must anticipate the opposite elastic transformation: self-weight concentrating at the abutments rather than at the crown. The resulting compressive catenary will be fuller at the apex than its plain cousin rather than sharper, and it will be steeper at the bases. While the axial strain of physical catenaries is very real, the materials used in arch building are pretty rigid, and the structures are stiffened against small irregularities. So the contradiction between tensile and compressive deformation can safely be ignored. However, in the design of large tensile spans (especially 
when using stretchy polymers) and for the completeness of our mental models, axial strains should probably be acknowledged.

It is also worth noting that neither of the axially strained catenaries is particularly desirable in structural design. They both entail redundant material at mid-span and over-stressed material at the anchorages. Nor, for that matter, is the much-vaunted plain catenary all that efficient. It, too, contains dead weight in the middle and overtaxed material at the ends. Whether extruded by a spider or spun in a cable factory, the constant cross section of the drooping element reflects the convenience of making and the largeness and unpredictability of live loads, not an optimal distribution of self-weight. Of course, designers are free to re-distribute material so as to achieve the more truly optimal equally stressed catenaries. Both in suspension and compression spans, the cross section and self-weight per arc length would increase toward the ends in proportion to increasing axial loads. Rather like the compressive catenary, both curves would be steeper toward the ends and fuller toward mid-span than the plain catenary. A rough illustration may be found in the well known Jefferson Memorial or Gateway Arch. The precise geometry of Saarinen's arch is controversial, but its downward widening, as well as providing moment anchorages against wind loads, can be understood as accommodating accumulating self-weight. In a material like steel whose tensile and compressive behavior is symmetrical, the compressive and tensile versions of the equally stressed catenary would be identical. However, unlike the plain and elastic catenaries, this equally stressed curve cannot be found by simple mechanical means.

In the plain catenary, the desired condition of zero bending stresses is congruent to a condition of least potential energy or lowest center of gravity. So, allowing a chain to sag to the low-energy state happens to achieve or to "find" the desired form. It is hard to imagine a mechanism that would sag or otherwise "wind down" to a condition of equal stress. What the form-finding mechanism would need to do is to move material uphill, from mid-span toward the anchorages. This could be done by iteratively measuring strain, modifying the structure, and allowing it to adopt a new form, but this process is much more involved than simply hanging a chain. The complex mathematics of equal stress funiculars has been set out by Williams (2014: 28-31). But whether achieved on site, in a laboratory, or through a mathematical model, the optimal distribution of material would appear to be a work of artifice and not of nature.

Yet again, this sharp opposition of natural and human might be spurious. What distinguishes the stress-equalizing processes of iterative design, testing, and making from the closed system of the hanging chain is, first, the introduction of energy needed to move material, and second, the information processing needed to redistribute material appropriately. In this respect the hanging chain, like the soap bubble or the drafting spline, can be seen as an entropic system. The measure-andrespond processes of stress equalization, by contrast, are negentropic. Bone, muscle, and other tissues can inherently distribute material so as to reduce stress differentials, just as engineers can. So a distinction emerges between entropy on the one hand-mechanically based - and negentropy on the other hand-whether biologically or mentally based. In the world of form-finding this distinction may be more appropriate than an exaggerated opposition of nature and artifice. This is not 
idle hypothesis. Very possibly, the living root bridges of Meghalaya apply some degree of negentropic stress-equalization to their funicular structures.

\section{Some Other Funiculars}

Curved, free-standing arches are comparatively rare in architecture and engineering: much more common are segmented arches carrying a superimposed or suspended deck. To achieve something like least weight, the trajectory of such arches must reconcile the parabolic funicular of the continuous deck, the exponential funicular of the lengthening hangers or piers, and the equally stressed funicular of the arch itself as it accumulates self-weight. As a matter of interest, where a funicular arch is segmented between hangers or piers, it will be often easier and always more accurate to calculate slopes, dimensions, and masses incrementally rather than as an analytic approximation. So, while we did not build such a "viaduct arch", it was a relatively simple matter to build a spreadsheet that progressively computed the weight and slope of the funicular segments downwards from an arbitrarily weighted and sloped pair of segments at the crown.

Sometimes an arch "wants" to follow a plain catenary centerline in spite of a varying section, as in the case of the two-hinged masonry arch shown in Fig. 1. Here, the voussoirs were made hollow for efficient employment of mass, and their width narrows exactly as their depth increases-rather in the manner of Eiffel's bridge arches. Constant perimeter, reasonably equal arc length, and negligible transverse stiffening translate closely enough to a constant distribution of mass along the arc that the catenary trajectory made sense.

Rather than pruning structures toward material economy, we can redistribute self-weight to reflect formal purposes. For example, a chain can be suspended and weighted to fall on any circular arc less than $180^{\circ}$. In one of our experiments, we

Fig. 1 Hollow blocks of this two hinged masonry arch are of constant transverse perimeter and close to equal weight; thus follow a plain catenary trajectory

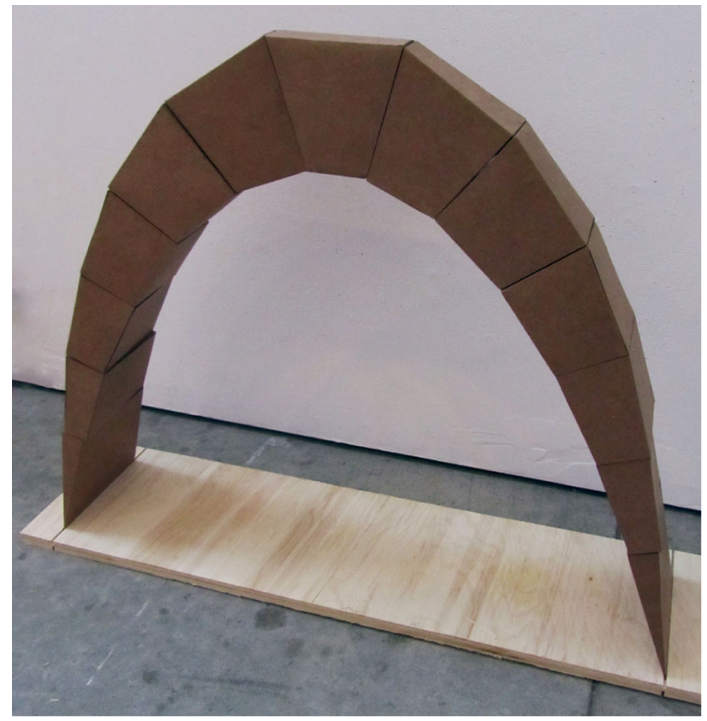


translated those weights into equivalent "lengths" of voussoir measured and cut from double-beveled strips of wood. The constant bevels ensure a circular arc, while the lengths of the voussoirs increasing toward the base made the requisite distribution of mass graphically legible. We call this structurally expressive arch an arc-weighted catenary, illustrated in Fig. 2a. The plain catenary responds precisely to an arbitrarily uniform section and as such is conventionally celebrated as an ideal. But our arch, precisely weighted to an arbitrary profile, is equally reflective of its own quantitative pretext. Furthermore, the sigmoid plan provides at least some equalization of axial stresses between base and crown, and it provides much-needed stability across the plane of the arch. So conceptually the structure is just as "ideal" as a plain catenary arch while pragmatically it has structural advantages.

Another arch (Fig. 2b) was designed to fall on a double-triangle or bow-tie plan; here the weights, centroids, forces, components, and angles were calculated downwards, block by block, always seeking constant horizontal thrust, using a spreadsheet similar to the viaduct calculator mentioned above (Jannasch 2016b). The taper is not as interesting as in the arc-weighted catenary, but it provides better equalization of stress and transverse resistance. This arch we termed a lunar catenary, for reasons that will become clear later, in the discussion of domes.

\section{Counter-Arch as Counter-Example}

We termed one arch-like structure the counter-arch, because its concave outlines and especially its notched keystone disguise and contradict the trajectory of forces. The static success of this arch demonstrates just how far the intrados and extrados of a moderately thick freestanding arch can depart from the funicular, even as the voussoir joints respect it. It illustrates a mannerist and self-conscious approach to structure. We might even call the form unnatural, in that it could not likely be found by mechanical or even biological means, but only through more complicated processes involving the human imagination. It is important to notice that the
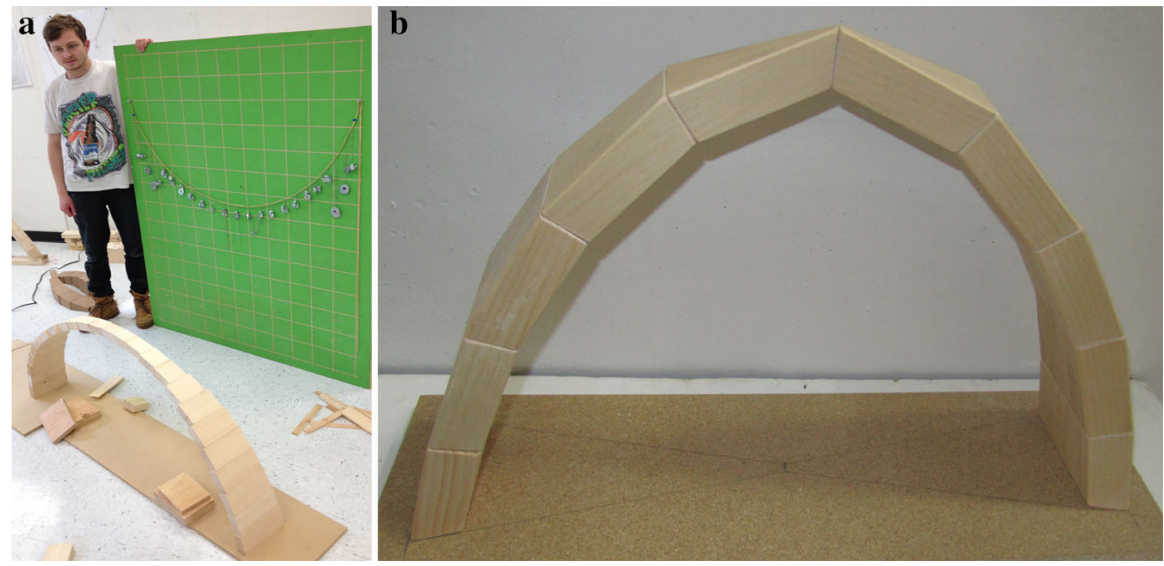

Fig. 2 a Catenary arch weighted to form circular arc; $\mathbf{b}$ catenary arch formed to a bow-tie plan 
working principles of the counter-arch are quite different from those embodied in the bell-shaped pseudome discussed later, which succeeds even when the structure is very thin and the funicular does not pass through the joints.

\section{From Arch to Dome}

\section{Some Definitions and Constraints}

Usage varies, even among specialists, so it is worth pausing to define what we mean by dome and by masonry. We called all of our surface structures domes, even when they depart from conventional forms, because they maintain the dome's essential symmetries. They are all wholly circular, without openings, corrugations, or other interruptions. Continuous rotational symmetry means that meridional action is everywhere identical, and that the meridional action in a radial plane is easily abstracted from hoop-wise action in the horizontal planes. Finally, our domes include tension hoops only at their bases. Domes defined in this way represent a tiny subset of the wealth of possible vault and shell forms, but this constraint allowed a more focused study of structural behaviors and of formal possibilities. Within this realm of domes we named important variants as we encountered them: our qualifying terms will be explained as they arise in the text.

By masonry we mean un-mortared horizontally coursed unit masonry. We distinguish between butt joints, which always fall on radial planes, and bedding joints, which always fall on conical surfaces. Unit masonry exhibits low tensile capacity across the joints so a horizontal course resists little hoop tension and a vertical section is quite free to develop hinges. By excluding any kind of mortar or glue we ensured that tensile strength across joints was zero and thus resistance to bursting and hinging would be minimal. Eliminating adhesion also kept the shear resistance along joints very low, especially where printed ABS blocks were used. This emphasized any tendency of the blocks to slide inwards, for example, or to be ejected outwards. Compared to laminated, hexagonal, or other complex tiling patterns, the continuous horizontal coursing offers minimum resistance to hinging. Both the lack of adhesion and the continuous coursing emphasize the failure modes of unit masonry-so that we could better appreciate its characteristic strengths.

\section{Poleni}

Giovanni Poleni extended Hooke's idea of the hanging chain to the analysis of domes. The critical difference between arches and domes is explained in plates XII and XIII of his Memoire, given here in Fig. 3 (Poleni 1748). Poleni's Fig. XII illustrates Hooke's principle that the hanging uniform chain can be inverted into a standing uniform arch. Fig. XIII illustrates the difference between a half-arch of uniform section and a half-arch or "lune" sliced from a dome. In a lune, the self weight is not uniform, but diminishes at an increasing rate from an arbitrary amount at the base to zero at the apex. The funicular of a lune therefore traces a fuller curve 
Fig. 3 The joints of the bellshaped counter-arch contain its funicular
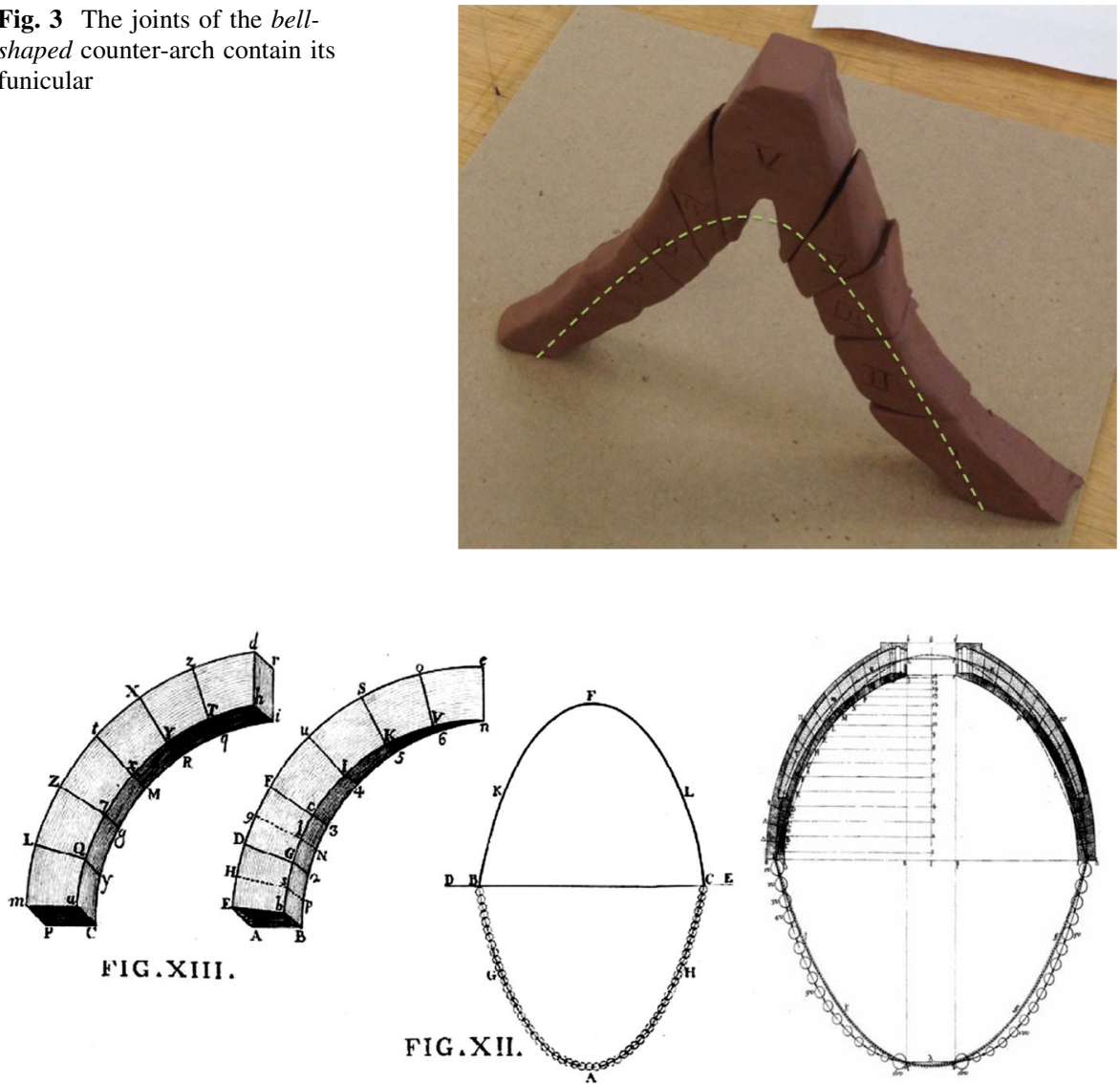

Fig. 4 Poleni's semi-arch and lune, his illustration of Hooke's dictum, and his weighted-chain analysis of St. Peters (from Poleni 1748)

than the funicular of a plain arch: it is steeper at the ends and shallower in the middle (Fig. 4).

In his funicular analysis of St Peter's, Poleni sized his weights to reflect not only the width of each lune increasing toward the base, but also the total thickness of the masonry shells as they varied along their meridian. The array is completed by heavy weights modeling the mass of the lantern and the absence of weights in the oculus. The analysis provided by this mechanism is straightforward and accurate. But the device cannot be applied directly to the design of a funicular dome.

To derive the funicular of an even-thickness dome from a hanging chain requires that each link be weighted to its circumferential extent, or thus to its distance from the vertical axis-but this distance would change with every re-weighting of the chain. Equalizing the axial stress becomes even more complicated, as each link of the chain must be iteratively measured, weighted and adjusted to account for both stress and circumferential extent. For a mathematical approach to this complexity, the reader is again referred to Chris Williams' treatment (Williams 2014: 28-31). 


\section{Gaudí}

That a structural form cannot break the laws of nature, that it should appear to express rather than disguise or contradict the actions of nature, and that it can be found through material rather than abstracted means is epitomized in the work of Antoni Gaudí. The idea of allowing inanimate nature to find structural forms can be as compelling as the structures themselves, so images of Gaudí's hanging models may play a greater role in our structural imagination than the resulting buildings. But these models are neither as complete nor as certain as their scale and complexity suggest. As we saw earlier, hanging chains do arrange given members into a zerobending state, but they cannot size the members. Members in a chain or a net of chains must be sized in response to loads by some mix of guesswork and calculation. Some of these loads, such as the extent of surface supported by particular ribs, can only be approximated after a rib has found its position. Reweighting the chains according to the spaces found between them would involve long iterations of re-measurement and re-adjustment. If an entire surface were defined by hanging chains, a completely funicular shell would have been achieved, with no loads or actions acting across the funicular arcs. But now the chains themselves would have to be made thicker and thinner to fill the space between their centerlines. The form of a funicular dome could be closely approximated in this painstaking manner, and doing so would surely be a remarkable achievement. But the one-way structural grain implied in this method would not begin to exploit the two-way capacities of shell constructions. So even if the smooth funicular dome were in some limited sense seen to be a "natural" form, it would not really express or exploit the geometrical and structural nature of shells. And unlike the linear catenary, it probably doesn't exist in nature. Gaudí was a student of nature and he was interested in the in-plane shear capacities of laminated masonry vaults. So he scalloped and ribbed his shells to utilize these potentials. The configuration and proportion of these undulations was not determined by an omniscient chain, however, but by the inspired intuition of a structural artist.

\section{Dome-Finding}

The thinner an arch the more tightly it must fit its funicular and the more clearly it will express the nature of weight, gravity, and equilibrium in a vertical plane. When the design is traced from a hanging chain, bypassing mathematical abstraction, the form not only answers to nature but has been designed through the forces of nature. This double integration of the artifact with cosmos produces elegant forms, and it reassures builders of the rightness and perhaps even the righteousness of their approach. But as we have seen with Gaudí's methods, the transposition of the funicular across dimensions is problematic. Surfaces in space behave differently than curves in a plane. Sheets curve freely in one direction just as a line can, but not simultaneously crosswise. Soap films freely distribute material over a surface and are used in some form-finding exercises. However, the physics involved minimizes surface area, which has nothing to do with resisting gravitational loads. Even loosely woven, soaked fabrics suspended in a gravitational field behave differently 
when stressed diagonally and orthogonally, and while they do allow a degree of inplane shear transformation, they are not actually extensible. Stretchy materials embody the disadvantages both of tensile films and of fabrics. Consequently, if a suspended sheet is to achieve significant Gaussian curvature, it must be pre-tailored to a design. Nature can then find the form anticipated by the designer.

Deformation in one direction of a surface inhibits deformation in the crosswise direction. This is simply the nature of surfaces in space. So the funicular that is defined by a chain and that is essential in freestanding arches is impossible to achieve with sheet materials. But, for the same reasons, the funicular is not really required in shells. Curvature on one axis of a shell inhibits deformation in the other, so the surface effectively resists bending loads. Nature insists that a spider's filament hang in a catenary arc, but the nature of surfaces allows seed pods, sea shells, animal horns, and other thin structures to take a magnificent variety of forms optimized to many different purposes. Designers of stressed ribbon bridges and straight vaults are almost as constrained in their form-making as the spider, but in the design of ships' hulls, corrugated sheds, and monocoques of all kinds they are free to employ all the convolutions that maintain the form of grown and evolved shells. The self-stiffening nature of doubly curved shells is clearly evident in metal and reinforced concrete and other constructions that resist both tension and compression. But there is considerable freedom of form even in the design of fanciful hot-air balloons, in spite of their compressive weakness, and in masonry shells, despite their utter incapacity in tension. So the funicular profile defines only one of many possible masonry domes.

\section{Lunar Catenary and Funicular Dome}

The arch in Fig. 2b is a catenary formed to a bow tie plan. Intersecting several bowtie arches through their apex would create a polygonal dome. There could be no hoop action whether positive or negative either in the individual arches or in the dome. Each half-arch on its triangular plan is the lune of a dome; hence the name given earlier: the lunar catenary. The bow-tie arch is one diametral slice of a funicular dome, and only a funicular dome could produce a freestanding bow-tie arch.

What anyone building such a dome or even such an arch can report is that they are extremely fragile, especially as they get thinner. Even a tiny applied load will move the funicular outside the thickness of the masonry, resulting in immediate collapse. Fortunately, there is a whole realm of masonry domes that perform better.

\section{Four Hyper-funiculars}

\section{The Shallow Spheric Dome}

We learn from Heyman in The Stone Skeleton (Heyman 1995: 33-35) that if we trace the funicular of a uniformly thin hemispherical dome through its springing and apex, we'll find that the two curves intersect at an angular altitude of about $52^{\circ}$. Above this 
point, the masonry is within the funicular and below that point, it passes outside the funicular. As with the arch, this means that the lower parts of the dome will burst outward unless contained by tensile hoops, external buttressing, or some other means. But unlike the arch, the upper portions of the dome cannot fall inward, because the wedge-shaped blocks in each course press against each other forming a circular horizontal arch. The circular trajectory of thrust in each of these arches can be interpreted as a negative or compressive hoop stress. A shallow spheric dome exhibits these compressive hoop stresses throughout. It is therefore stable with respect to the self-weight emphasized in structural mathematics, and it is also pre-stressed against the uneven live loads of material reality. Such a dome is more stable than the funicular dome of zero hoop-stress, which, unless it is uneconomically thick, doubleshelled, or otherwise stiffened, is always on the verge of bursting.

If we visualize the domical funicular as a lune-weighted hanging chain, the section of an inverted shallow dome lies above the funicular. For this reason, and because such domes perform better than the funicular equivalent, we call them hyperfunicular. More rounded domes whose inverted section lies below the funicular trajectory we call hypo-funicular (and to reiterate: unlike domes, arches will fail in both non-funicular realms). Within the design space of hyper-funicular masonry domes it is hard to specify an optimal or ideal or natural form. As with organic structures, the hyper-funicular dome can adapt to many material and spatial demands.

\section{The Odd Case of the Catenary Dome}

Textbooks can be ambiguous about the action and form of domes, but we may sometimes infer that funicular domes follow the plain catenary of the uniform hanging chain. Our inference would be wrong, because as discussed earlier, the funiculars of a lune and of a half-arch are two different things. Nonetheless, a plain catenary would generate a successful masonry dome, because it falls within the fuller profile of the lunar catenary. In other words, the "incorrect" catenary dome would be hyper-funicular, and would perform better than its "ideal" or merely funicular counterpart.

\section{Wren's Cone}

Wren's brick cone at St. Paul's departs even farther from the funicular. Very clearly, an arch of this section would collapse inwards, but the conical structure succeeds. Looking at a section, it is not unreasonable to interpret the cone as the straightlegged response of a relatively light dome to a significant apical load. But Wren's thinking was more thorough than this. He understood that during every stage of the cone's construction, each circular course acted as a circular arch, and that the conical frustum was stable with or without the surcharge of the lantern. He also brought loads from the outer timber dome to several courses of the brick cone. He recognized that the cone would resist these concentrated loads, without having to introduce funicular knuckles at the point of application. We know that Wren and Hooke talked about many things, including Hooke's principle of inverted funiculars. What Wren took from and added to their discussions will never be known. Nor can 
we tell how, exactly, Wren's ideas evolved concerning the hanging chain, the semicubical parabola, or the funicular of a structure as complicated as St. Paul's. However, that he had some sense of hyper-funicular masonry is clear from the design and the success of his remarkable conical structure.

Wren perforated the cone with dozens of openings. These aren't always visible in section but they are prominent in the compound drawing shown in Fig. 5. At first our team felt that the openings would destroy the horizontal arch action of the affected courses. This seemed to argue against Wren's awareness of or interest in this aspect of the cone. But eventually we saw that instead of inserting conventional rectangular windows with arched heads, designed to carry vertical loads, Wren framed the openings with circular arches. These can carry both vertical and horizontal loads. As if to underscore the structural rationale of the round openings, Wren did use conventional openings higher in the dome. And here, exactly where the horizontal arches have been interrupted by vertical slots, the masonry compensates to form radial stiffening ribs (these also make the transition from the simple dome to the complex plan of the lantern). So, far from compromising the horizontal arches of which the dome is built, the circular openings both maintain and exemplify their structural behavior (Fig. 6).

\section{Pseudomes}

We can take the hyper-funicular idea beyond the cone into the anticlastic realm of tent- or bell-shaped domes. Mackenzie posited one such dome in his study of King's

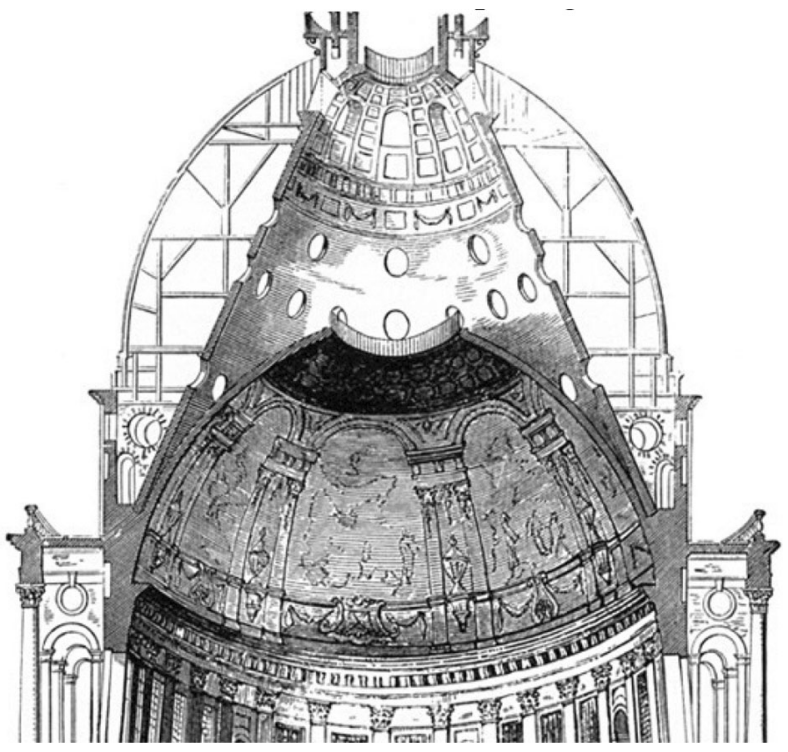

Fig. 5 Wren's conical dome at St. Paul's punctuated by circular arches where they carry horizontal as well as vertical forces, and by parallel side openings where they fall between independent ribs (from Dimmock Dimmock 1900) (Project Gutenberg) 

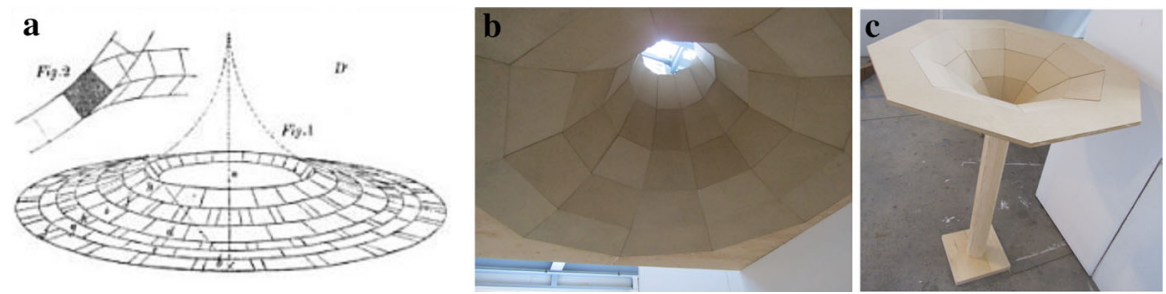

Fig. 6 a Mackenzie's anticlastic dome (Mackenzie 1840) (Google Books), b underside of $2.3 \mathrm{~m}$ diameter pseudome, c $0.5 \mathrm{~m}$ diameter fandome

College Chapel at Cambridge (Mackenzie 1840: 86). He did not expect it would be built; it was a heuristic proposal only, a theoretical inversion of the fan-vaults at King's drawn to help explain their structural behavior. Our team built two anticlastic domes. One was about $.5 \mathrm{~m}$ in diameter using $12 \mathrm{~mm} \mathrm{MDF}$, for a span to thickness ratio greater than 40:1. The second was over $2.3 \mathrm{~m}$ in diameter and used panels of $18 \mathrm{~mm}$ chipboard, for a span to thickness ratio greater than 120:1. The anticlastic surface required that the panels be beveled inward on the vertical joints and outward on the horizontal joints. In building the domes, we learned that the opposed taper made it difficult to dislodge the panels in either direction. This gave a distinct structural advantage over any synclastic or developable form, funicular or otherwise. When we want to highlight this virtue, unique to synclastic masonry, we describe the forms as radically hyper-funicular. We first encountered Mackenzie's drawing in The Stone Skeleton (Heyman 1995: 81-83). Somewhat confusingly, Heyman adds funicular lines to the drawing to show that the trajectory of meridional forces would not coincide with the anticlastic surface, and he comments that the fanvaulting at the chapel is filled with mortar and rubble to contain these forces. The observation and the remedial measure may be needed in the half-fans in the actual chapel itself, but they do not apply to the circular structure drawn by Mackenzie or in fully developed fan vaults, where hoop compression can contain the apparently "stray" funiculars, bypassing the unnecessary fill.

If there is an ideal form for anticlastic domes it has so far eluded us. One candidate is the hemi-pseudosphere. This is generated by rotating a half-tractrix about its longitudinal axis. Although the surface stretches to infinity on the vertical axis, it has the same volume as a hemisphere of the same radius, and constant Gaussian curvature. But whatever the geometric elegance of this inversion, it has no particular structural merit. The realm of radically hyper-funicular domes is large, and within this realm of stability the form may freely be optimized to other considerations. Nonetheless, for their affinity to the canonical pseudosphere, we refer to all the anticlastic domes as pseudomes. In our case, we wanted to simplify layout and fabrication, so we adopted quarter-circle profiles that generated torus patches. As with animal horns, sea shells, and other organic forms, the material and energy economy of mass is thus balanced against the informational economy of description and production (Jannasch 2004: 383-392).

In the spirit of Mackenzie's proposal, we cut the base panel of the small dome into a polygon, and inverted the structure onto a polygonal column. In light of its 
heritage, we called this structure the fandome. Superimposing a fandome on a pseudome would produce something akin to a hyperboloid cooling tower, perhaps earning the name enantiodome.

\section{Four A-Funiculars}

\section{The Corbelled Cone}

In funicular and hyper-funicular domes, the horizontal bedding joints fall on conical surfaces normal to the surface of the dome and to the meridional line of thrust. This configuration is not strictly necessary. In some Mediterranean cultures, circular stone roofs are built with bedding joints that clearly do not involve meridional compression forces. We describe these corbelled domes as a-funicular. The stones in these structures are typically quite flat, but we printed a set of ABS blocks that are twice as high as the horizontal thickness of the cone they create (Fig. 7a, b). This illustrates certain oddities of corbel behavior. The cone is inclined at a slope of 1:2, so in a straight block of the same section the center of gravity would fall directly over the heel of the stone, allowing the block to tip inwards at the least disturbance. With our blocks, however, curved to a conical form, the centroid is located over the plan of the base. This means that the blocks are stable in the upright position. The mechanics by which blocks in superimposed corbelled courses support each other are not straightforward, but we did not pursue them much farther. We had actually made the kit of blocks for another purpose.

\section{Cuneidomes}

The blocks were made to be used in the inverted orientation, with the cone pointing downward. Because the primary faces of the blocks all fall on the same cone, they also fall on concentric cones of the same slope. This allows the conical surfaces to be used as the bedding surfaces between courses (Fig. 7c). In a corbelled dome the bedding planes are shallower than perpendicular to the nominal line of thrust; here they are steeper. Each circular course forms something like a drain plug that cannot drop through its supporting course because of the wedge action-which also serves to squeeze the course more tightly together. In reference to the wedging action of
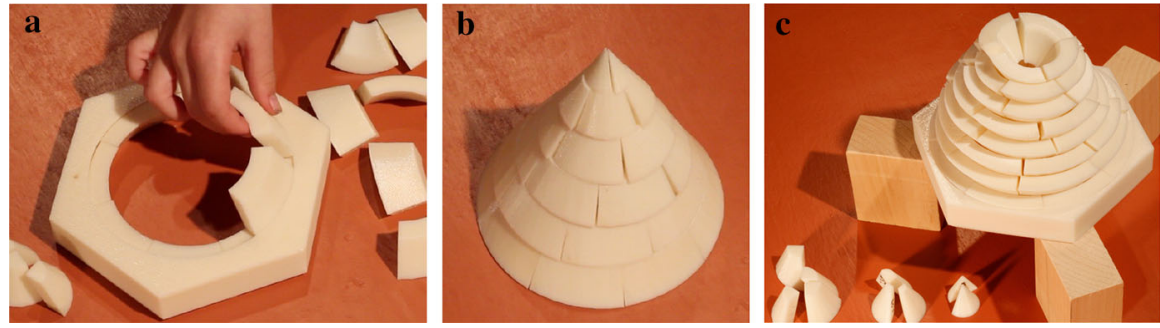

Fig. 7 a Corbelled cone under construction, b cone completed, c cuneidome almost complete 
the courses, we calling structures built in this way cuneidomes. Ours clearly illustrates the horizontal shear capacity of the blocks that acts over annular shear faces and that keeps the upper courses of this structure from bursting outward through the lower courses. This horizontal shear strength must be at work in all domes, though it is often taken for granted. As with the corbelled domes, lines of compressive force perpendicular to the joints can no longer be connected into a smooth trajectory, so we include cuneidomes among the a-funiculars.

\section{Flat Vaults or Floordomes}

If we remove every second course of the cuneidome shown above, the wedgeshaped courses settle to the same elevation to create a flat surface. Our version of what we named the floordome is very modestly proportioned (Fig. 8). Each block, as we know, is twice as high as it is radially deep, and the whole floor has a cautious span to thickness ratio of 5:1. This pales in comparison to Herrera's flat vault at El Escorial, which is almost $8 \mathrm{~m}$ in diameter and only $0.285 \mathrm{~m}$ thick, for a ratio of $27: 1$ (Addis 2007: 157). Furthermore, the blocks each extend almost twice as far radially as in thickness, so they seem vulnerable to rotating in the radial plane. Addis characterizes this floor as a two-way spanning arch, but the section clearly would not work as a straight arch or even as a spider of intersecting arches. So other actions must be involved. One of these is the vertical shear acting over concentric cylindrical faces, transferring vertical loads outward. Another is the ring compression concentrated in the upper and inner portions of each block, locking each course into a conical wedge, and restraining the blocks from rotating downward about the toe of the supporting course.

These capacities are also at work in conventional domes, the first preventing higher parts of a dome from crashing through the lower parts, and the second preventing individual blocks from rolling inward. As with the horizontal shear illustrated in the cuneidome, domes seldom fail in these modes. So it is no disaster to ignore them. Nonetheless, ignorance of these actions may limit our ideas about the nature and potential of masonry.

It is also worth observing that while Herrera's floor is a masterpiece of stonecutting, it is possible to build flat vaults or floordomes of the coarsest
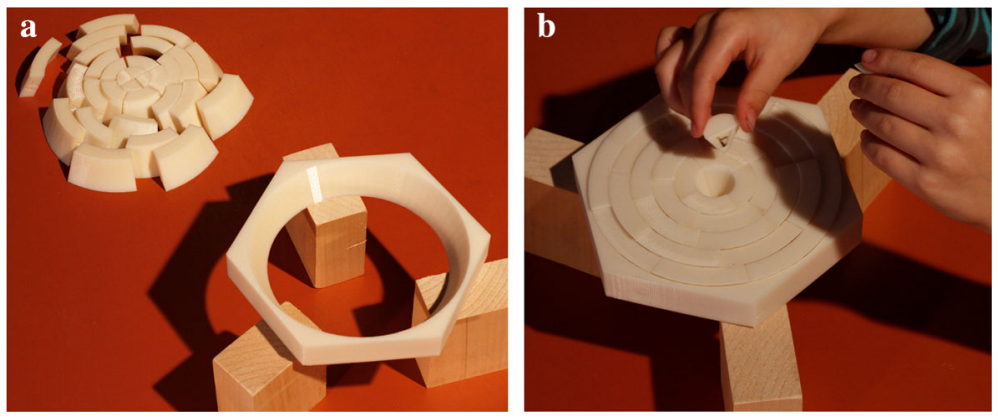

Fig. 8 a Floordome ready for construction, b floordome almost complete 
components. Meera Prajapati has built several, using plain brick and dry, loose gravel. The process is shown in Tanya Pahwa's on-line video (Pahwa 2012).

\section{Antidomes}

Spacing the courses of the floordome radially allows them to settle into a stepped V. These inversions of the normally rising dome we named antidomes. The example shown in Fig. 9a, b steps down about half a vertical thickness per course. The steps need not be articulated: Fig. 9c shows a smooth antidome. This is in fact our first experiment: more dramatic but less didactic than the stepped versions.

In Fig. 10, a floordome and two antidomes are drawn in cross section. It is clear that these structures could not succeed as arches; that they are not merely omnidirectional arches. Their actual behavior takes a little unraveling, which is best started with Fig. 10a, the floor-dome. Here, the broken red lines indicate cylindrical vertical shear surfaces. The outer shear surface crosses a bedding joint, the inner one falls within a single block. We observe that if the vertical load of each course is transmitted to its supporting neighbor in a direction normal to the bedding joint, we cannot connect these vectors into a simple funicular trajectory. We must first resolve them into components of vertical shear and horizontal thrust. In Fig. 10b, the shallow anti-dome, the broken red line shows that the innermost blocks are fully beneath the outermost course. This tells us that an annular shear surface has emerged across courses 2, 3, and 4 . We can imagine vertical tension beginning to act across this surface as well, accumulating toward the abutment ring. Figure 10c shows a steeper anti-dome and here the broken red line shows that each block is now fully above or below its next-to-neighboring counterpart. The idea looks entirely ludicrous. It seems that the stones must simply rotate inwards and drop down. But in three-dimensional space this can not happen. As in shallower anti-domes, the tendency of a stone to slide downward and inward is resisted by the hoopcompression that ties that course into a single wedge. And the tendency of any block to rotate about the toe of the supporting course is resisted by the hoop-compression in its upper portions. The red line also shows that in courses 2 and 3 horizontal shear and vertical tension are now acting explicitly, within individual blocks.

These structures are singularly un-useful, but they are intriguing, and they ask us to consider non-funicular actions at work in any dome such as hoop compression,
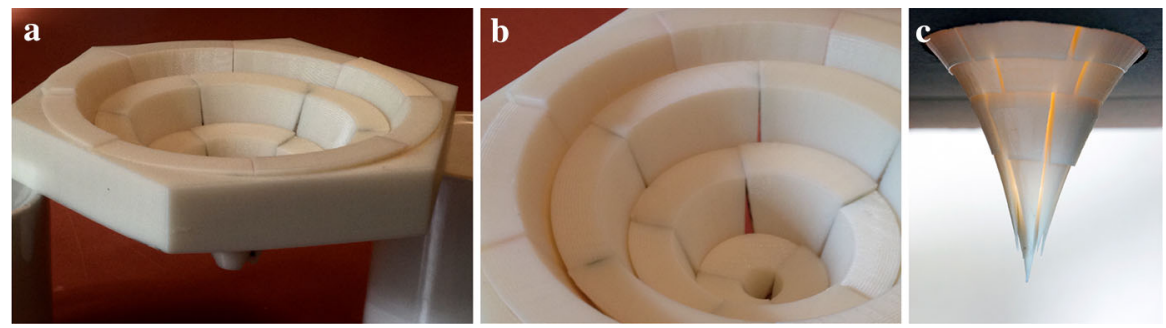

Fig. 9 a Low angle view of steep anti-dome, $\mathbf{b}$ higher angle view of steep antidome, $\mathbf{c}$ pseudospherical anti-dome 
Fig. 10 a floordome, b shallow antidome, c steep antidome
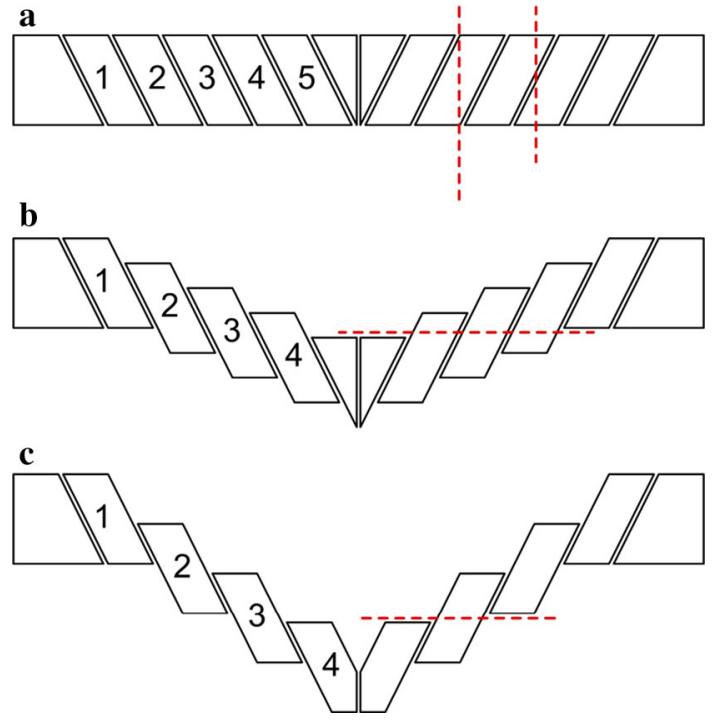

resistance to horizontal and vertical shear, and the consequent rotational resistance preventing blocks from rolling inward.

\section{The Case of the Ambidome}

The last structure described in this paper is the ambidome. In this project, a dome rises smoothly through its first courses in the usual manner but then transforms gradually into an anti-dome, descending from a circular crown to an oculus (Fig. 11). The structural transition does not happen suddenly either. It begins with the very first course, because as we assemble the slippery blocks we are reminded that what the structure depends on is not the unfinished and illusory meridional arches, but the complete and functional horizontal arches as they are installed. This was our premise at the outset of the whole adventure. The ambidome makes it abundantly clear that each course is in significant compression, and that even in the
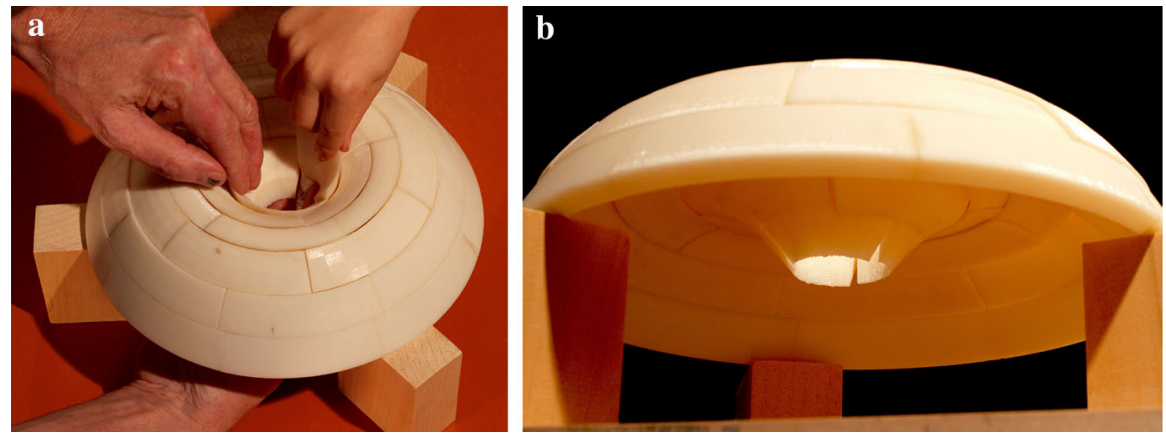

Fig. 11 a Ambidome under construction, b ambidome complete 
first, "normal" course, this compression enables all the secondary structural actions described above.

Testing our initial premise taught us more about masonry structures than we had anticipated. This experience in turn leads us to approach all structural theory with greater circumspection. And then, some of us came to admire the ambidome, for the counterpoise it establishes between explicit annular shelter and implicit vertical axis. In this light, the exercise was architecturally productive as well.

\section{Conclusion}

Physical trials of unusual masonry domes sharpened our understanding of masonry shells in general. Compression-only structures are not of great interest in the industrial world, but in regions where tensile materials remain costly and large-scale employment is desirable, a better grasp of masonry design is of immediate human value. This is especially true in the large areas of overlap with regions of seismic risk. But even for us, living well outside both realms, the experience of making these domes led us to challenge, sharpen and enrich our overall grasp of structural performance.

Accordingly, we recognize the special limitations of the dome form. As shells become pierced with doorways the depth of curvature in the remaining structure rapidly diminishes and here the funicular trajectory becomes extremely important, whether contained in the masonry itself or in voids between ribs or within lobes. Exploring what these lobes might look like in simply coursed, un-mortared masonry becomes an intriguing project.

Concerning design process, we contemplated material form-finding methods in the tradition of Gaudí and Otto, considering both their cultural importance and their material limitations. Over time, the difference between entropic and negentropic form-finding seemed to make more sense than an arbitrary boundary between artifact and nature. The new distinction brought to mind Bateson's account of the two realms of the Gnostic universe (Bateson 1979: 7). In this view, the inanimate and determinate pleroma is different from-but nevertheless gives rise to- the organic and information-processing creatura.

We also had time to consider the objectives of structural design. We began to ask ourselves what it is, exactly, that we identify as ideal, as optimal, or as functionally fit, and what we mean by formal freedom. When we celebrate a form as ideal, are we simply acknowledging our own discomfort with the roughness and complexity of nature? When we set out to optimize something to several explicit objectives, are we just trying to expand our ideals without threatening their essential abstraction? When we want to fit a structure to its anticipated function, how do we describe fitness - as a high span/depth ratio? Or a low self weight? Or should other affordances, costs, and expressions be accounted for? And what is freedom of form? The capacity to design and fabricate more complicated curves than spheres or cones or some other forms arbitrarily designated as un-free? The definition of un-free seems to be expanding over time, so that the converse sense of freedom is constrained by technology. In effect, free form in this sense means "definable 
exclusively by the latest curve-defining instruments but not by other methods." Be that as it may, why are the so-called free forms limited to smooth assemblages of higher order curvatures? Ultimately, any form or type is identifiable and constrained to its own identity, so the very notion of free form begins to look like an oxymoron. Nonetheless, we feel that a cogent sense of formal freedom can still be defined. To us, the freedom of form in shell structures is the inherent freedom of surfaces to take all manner of doubly curved forms; forms whose sections would fail as arches or as extruded into straight vaults. The forms we built within this realm drew on many kinds of geometries: conical, faceted, spherical and toroidal, and they extended to higher order curves and even hand-drawn shapes. Despite this formal liberty, all of our shells performed better than the ideal, notionally fit, and meticulously optimized funicular dome.

\section{References}

Addis, Bill. 2007. Building: 3000 Years of Design Engineering and Construction. London: Phaidon Press. Bateson, Gregory. 1979. Mind and Nature. New York: E.P. Dutton.

Dimmock, The Rev. Arthur. 1900. The Cathedral Church of Saint Paul: an Account of the Old and New Buildings With a Short Historical Sketch. London: George Bell and Sons.

Heyman, Jacques. 1995. The Stone Skeleton: Structural Engineering of Masonry Architecture. Cambridge: Cambridge University Press.

Jannasch, Emanuel. 2004. The Morphology of Artifact. In: Mathematics and Design 2004, Proceedings of the Fourth International Conference on Mathematics and Design, ed. Vera de Spinadel, 382-393. Buenos Aires: Universidad Tecnológica Nacional.

Jannasch, Emanuel. 2016a. Beyond the Funicular. In: Structure and Architecture: Beyond Their Limits, Proceedings of the Third International Conference on Structures and Architecture, ed. Paolo J.S. Cruz, 209-210. Leiden: CRC Press/Balkema.

Jannasch, Emanuel. 2016b. Teaching Form-Active Design with Lower Mathematics. In: Structure and Architecture: Beyond Their Limits, Proceedings of the Third International Conference on Structures and Architecture, ed. Paolo J.S. Cruz, 241-242. Leiden: CRC Press/Balkema.

Mackenzie, Frederick. 1840. Observations on the Construction of the Roof of King's College Chapel, Cambridge. London: John Weale.

Pahwa, Tanya. 2012. How to Make a Brick Roof. https://www.youtube.com/watch?v=cS1MABTq6Mk. (Accessed 31 May 2016).

Poleni, Giovanni. 1748. Memoire Istoriche della Gran Cupola del Tempio Vaticano. Padova.

Sandaker, Bjorn, Arne Eggen, and Mark Cruvellier. 2011. The Structural Basis of Architecture. Abingdon: Routledge.

Williams, Chris. 2014. What is a shell? In: Shell Structures for Architecture: Form Finding and Optimization, eds. Sigrid Adriaenssens, Philipe Block, Diederik Veenendal, and Chris Williams, 21-32. Abingdon: Routledge.

Emanuel Jannasch studied architecture at Cornell in the seventies and at Dalhousie in the nineties, where he earned his M.Arch. He has worked in carpentry and allied trades as well as in architectural offices, and has addressed unusual structural problems in marine and naval design, film and theater. He has published on prefabrication, architectural geometry, morphology, and built heritage, and has taught throughout Canada and abroad. He is currently Senior Instructor at the Dalhousie University School of Architecture where he has been teaching for 18 years. Questions of mind and nature he contemplates mainly through keeping bees. 